\title{
Transient behaviour and rate effects in resistive detectors
}

\author{
M.M. Fraga ${ }^{a}$, R. Ferreira Marques ${ }^{a}, *$, Y. Ivaniouchenkova, E.P. de Lima ${ }^{a}$, F. Neves ${ }^{a}$, \\ A.J.P.L. Policarpo ${ }^{\mathrm{a}}$, Carmen C. Bueno ${ }^{\mathrm{b}, \mathrm{c}}$, Josemary A.C. Gonçalves $^{\mathrm{b}, \mathrm{c}}$,

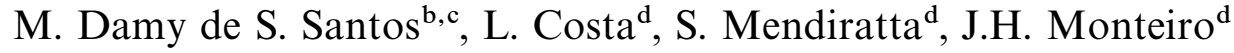 \\ ${ }^{a}$ Laboratório de Instrumentação e Física Experimental de Partículas, LIP - Coimbra, Departamento de Física, Universidade de Coimbra, \\ 3000 Coimbra, Portugal \\ ${ }^{\mathrm{b}}$ Instituto de Pesquisas Energéticas e Nucleares - Comissão Nacional de Energia Nuclear, 05508-900 - Caixa Postal 11049, São Paulo, Brazil

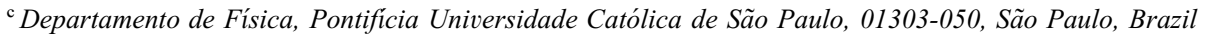 \\ d Departamento de Física, Universidade de Aveiro, 3800 Aveiro, Portugal
}

\begin{abstract}
The dependence of the detection efficiency (or charge gain) of a resistive detector on the counting rate has been considered by several authors. The influence of the resistivity of the electrodes, the counter geometry and the gas mixture composition have also been studied. Most of those measurements assume a stationary regime. In the present work we report on studies of the transient behaviour of detectors with a resistive cathode for various counting rates, charge gains and detector geometries. We show that in some cases the time-decay curves can be fitted by a single exponential plus a constant term, while in others (higher charge gains or higher counting rates) a sum of two or even three exponentials plus the constant term is needed to fit the experimental data. A study of the electric properties of the dielectrics used is also presented and a comparison is made between these results and the data obtained under irradiation conditions. (C) 1998 Elsevier Science B.V. All rights reserved.
\end{abstract}

PACS: 29.40.Cs; 29.40.Gx

Keywords: Proportional counters; Gaseous detectors; Resistive electrodes

\section{Introduction}

The effect of counting rate on the efficiency (or gain) of resistive detectors has been reported for a wide range of operating conditions and is normally attributed to the reduction of the effective

\footnotetext{
* Corresponding author. Tel.: + 35139833465 ; fax: + 35139 822358; e-mail rui@filip3.fis.uc.pt.
}

voltage across the gas gap, according to the equation $V_{\text {eff }}=V_{0}-R I$, where $V_{0}, I$ and $R$ are the applied voltage, the operating current and the electrode resistance, respectively [1-3]. In most cases, the studies were performed assuming a stationary regime and only a few authors refer to the dynamic behaviour of the detectors $[2,4]$. This effect is particularly important for spark mode operated RPCs, where a small area around the discharge spot remains inactive during a given period of time, thus 
limiting their rate capability. Some authors $[5,6]$ conclude that this dead time is of the order of $\rho \varepsilon \varepsilon_{0}$, where $\rho, \varepsilon$ and $\varepsilon_{0}$ are the bulk resistivity, the static dielectric constant and the permittivity of vacuum, respectively, and treat the dielectric as equivalent to a simple RC circuit. We used this approximation, in a previous work [7], to predict the transient behaviour of a proportional cylindrical counter with a glass tube as cathode, working under an irradiation rate for which the "mean effect" due to consecutive events results on a "current dependent" behaviour. The resistivity was measured during the test and for the dielectric constant the value given by the manufacturer was considered. The variation of the pulse amplitude with time was measured and compared with that expected from calculations. It was found that both curves could be fitted by a sum of two exponentials plus a constant term that corresponds to the pulse amplitude at equilibrium, but the experimental time constants were about one order of magnitude larger than those computed. Electric measurements of Crotty et al. [2], showed that the discharge of a phenolic sheet did not follow a single exponential decay law, and that the time constants were also larger than expected.

In order to clarify the role of the $\rho \varepsilon$ value on the relaxation of the detectors it was decided to study the electric properties of the materials and further investigate the relaxation mechanisms under irradiation conditions.

\section{Experimental setup}

The experimental data were obtained with two different detectors: a cylindrical counter and a parallel plate chamber, both operated in the proportional mode. The cylindrical counter, similar to the one described in previous papers $[7,8]$, consists of a glass tube $\left(\phi_{i}=12.7 \mathrm{~mm}\right)$, supplied by Schott, with a stainless-steel anode wire stretched along its axis. The parallel plate chamber is represented in Fig. 1. The anode is a common window glass plate, manufactured by Saint-Goban (COVINA).

The gas mixtures, $\mathrm{Ar}+10 \% \mathrm{CH}_{4}$ and $\mathrm{Ar}+20 \% \mathrm{C}_{2} \mathrm{H}_{6}$ at atmospheric pressure, flow continuously, at room temperature, through the cylindrical and the parallel plate chambers, respectively.

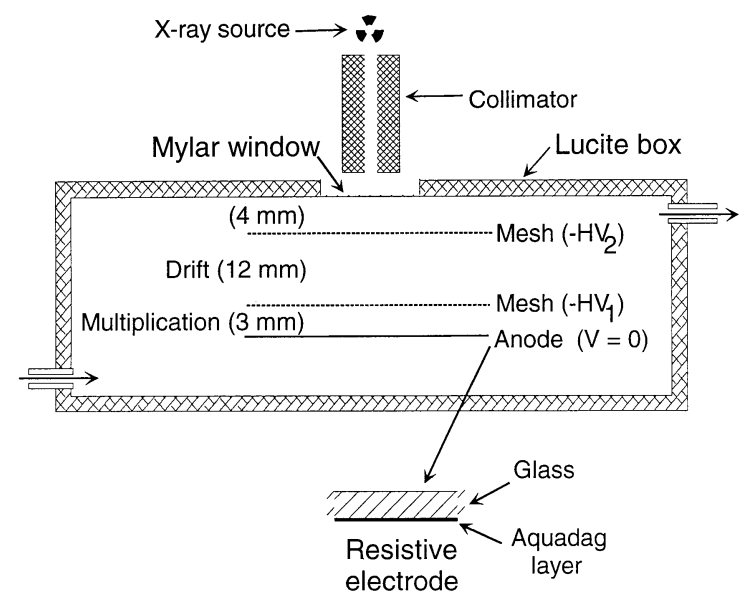

Fig. 1. Schematic view of the parallel plate chamber.

The cylindrical counter was irradiated, through the glass wall, by $22 \mathrm{keV} \mathrm{X}$-rays. The counting rate was changed by interposing aluminium foils of different thicknesses between the source and the detectors.

The charge gains were measured using either a conventional charge amplifier electronic system and a multichannel analyser or an electrometer. For the study of the transient effects, the signals from the amplifier are fed into an CAMAC ADC and then recorded by a computer.

The electrical properties of the glasses used were measured using small pieces cut from both the tube and the plate. Aluminium electrodes of $5 \mathrm{~mm}$ diameter were deposited by evaporation on the two sides of the ultrasonically cleaned samples, and the contacts were made of tinned copper wire with silver paint.

\section{Electrical measurements}

The $V-I$ characteristics of all the samples were measured up to $1000 \mathrm{~V}$, and all the samples showed an ohmic behaviour. The resistivity values at room temperature are listed in Table 1 . The variation of resistivity with temperature investigated in the range of $0-70^{\circ} \mathrm{C}$, showed an Arrhenius behaviour [9]. A typical dependence of $\varepsilon^{*}\left(\equiv \varepsilon^{\prime}+i \varepsilon^{\prime \prime}\right)$ on frequency is shown in Fig. 2, for the glass plate. Similar results were obtained for the glass tube. The 
Table 1

Characteristics of the two glass electrodes

\begin{tabular}{lcl}
\hline & Glass tube & Glass plate \\
\hline Thickness & $0.7 \mathrm{~mm}$ & $2 \mathrm{~mm}$ \\
Resistivity $(\Omega \mathrm{cm})$ & $3.62 \mathrm{E}+12$ & $5.18 \mathrm{E}+12$ \\
Dielectric constant & 10.7 & 9.4 \\
$\rho \varepsilon \varepsilon_{0}$ & $3.4 \mathrm{~s}$ & $4.3 \mathrm{~s}$ \\
\hline
\end{tabular}

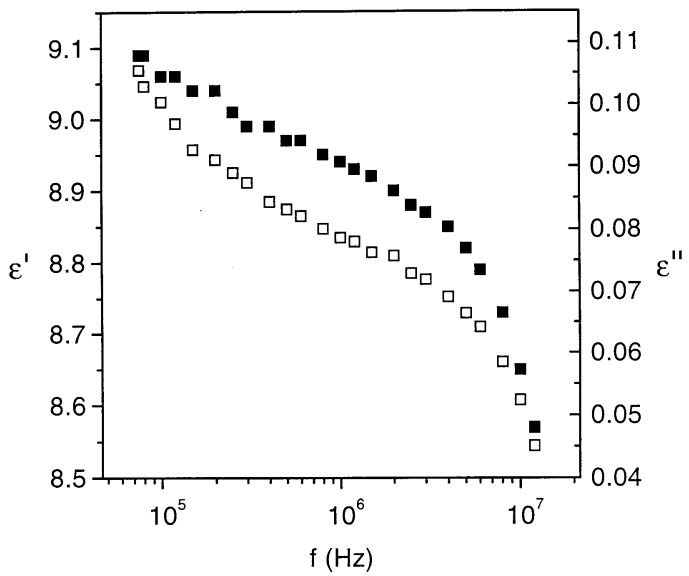

Fig. 2. Variation with the frequency of the real (solid squares) and imaginary part (open squares) of the dielectric constant of the glass plate.

values of the low frequency (or quasi-static) dielectric constant are also indicated in Table 1.

A tool to understand some aspects of the glass structures is the measurement of the dynamic behaviour of the depolarisation current, which is directly related to the relaxation function of the dielectric, $\phi(t)$, by $I_{\mathrm{D}}=\mathrm{d} \phi / \mathrm{d} t$. We measured that current over a long period, starting as close as possible to the switching-off of the applied electric field. A typical $I_{\mathrm{D}}(t)$ curve is shown in Fig. 3, for the glass plate, for an applied voltage of $100 \mathrm{~V}$.

As it can be seen from the figure, $I_{\mathrm{D}}(t)$ is not a single exponential function as it would be expected for an ideal dielectric. In fact, for glasses more than one process is responsible for the delayed polarisation and, in a first approximation, we consider each of them to be characterised by a separate relaxation time, $\tau_{i}$. $\phi(t)$ is then given by a sum of exponentials [9]. However, due to the complexity of the glass structure, there is a wide

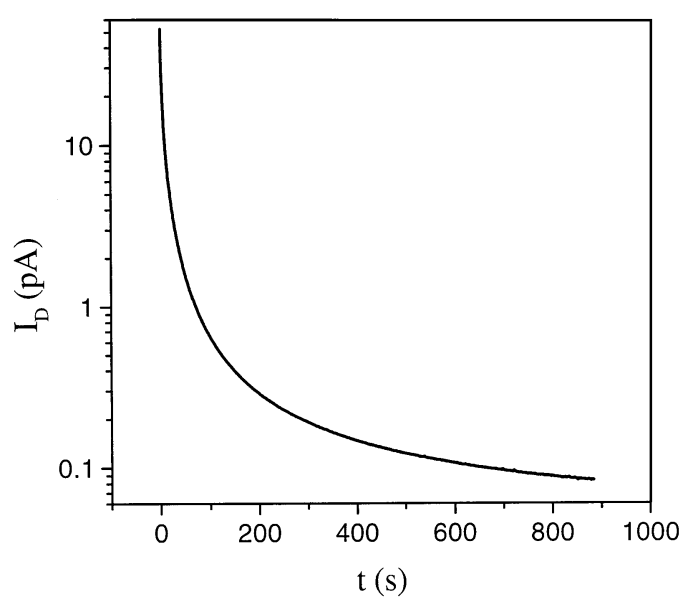

Fig. 3. Depolarisation current as a function of time.

Table 2

Characteristic parameters of the time decay of the depolarisation current

\begin{tabular}{lllll}
\hline & \multicolumn{2}{l}{$\phi(t)=\exp \left(-\left(t / \tau_{k}\right)^{\beta}\right)$} & \multicolumn{2}{l}{$\phi(t)=\Sigma A_{i} \exp \left(-t / \tau_{i}\right)$} \\
\hline & $\tau_{k}(\mathrm{~s})$ & $\beta$ & $\tau_{1}(\mathrm{~s})$ & $\tau_{2}(\mathrm{~s})$ \\
\cline { 2 - 5 } Glass tube & 22 & 0.144 & $2.9 \pm 0.1$ & $33.7 \pm 0.3$ \\
Glass plate & 13 & 0.171 & $2.7 \pm 0.1$ & $25.2 \pm 0.9$ \\
\hline
\end{tabular}

range of mutually interacting processes and $\phi(t)$ follows the stretched exponential law, $\phi(t)=\exp \left(-\left(t / \tau_{k}\right)^{\beta}\right) \quad[10]$, where $0<\beta<1$ is a parameter describing the shape of the time dependence of the transient current and $\tau_{k}$ is an effective relaxation time. Details about the fitting procedure are given in Ref. [10]. The resulting $\tau_{k}$ and $\beta$ values for the glass tube and the glass plate are listed in Table 2. In a rough approximation we can consider only the first $200 \mathrm{~s}$, so that such data can be compared with the results under irradiation, and assume a two exponential decay. The resulting time constants, also listed in Table 2 for comparison, are larger up to a factor of 10 or of the order of $\rho \varepsilon \varepsilon_{0}$.

\section{Results under irradiation}

Due to the finite conductivity of the resistive electrode, as soon as the high voltage is applied, 
and in the absence of the irradiation source, a current starts to flow across the dielectric. When the stationary state is reached, i.e., upon relaxation of the dielectric, the internal surface of this electrode is at the same potential as the external conducting Aquadag layer (grounded). If, starting at $t=0$, proportional counter pulses are produced in the detector, at a mean rate $v$, and with a mean charge per event $Q(t)$, a current $i_{\mathrm{g}}(t)=Q(t)$.v begins to flow through the gas. This results in an increase of the potential of the internal surface of the dielectric, i.e., in a decrease of the potential across the gas gap and, consequently, of the charge gain. Therefore, $i_{\mathrm{g}}(t)$ decays with time and tends to a constant
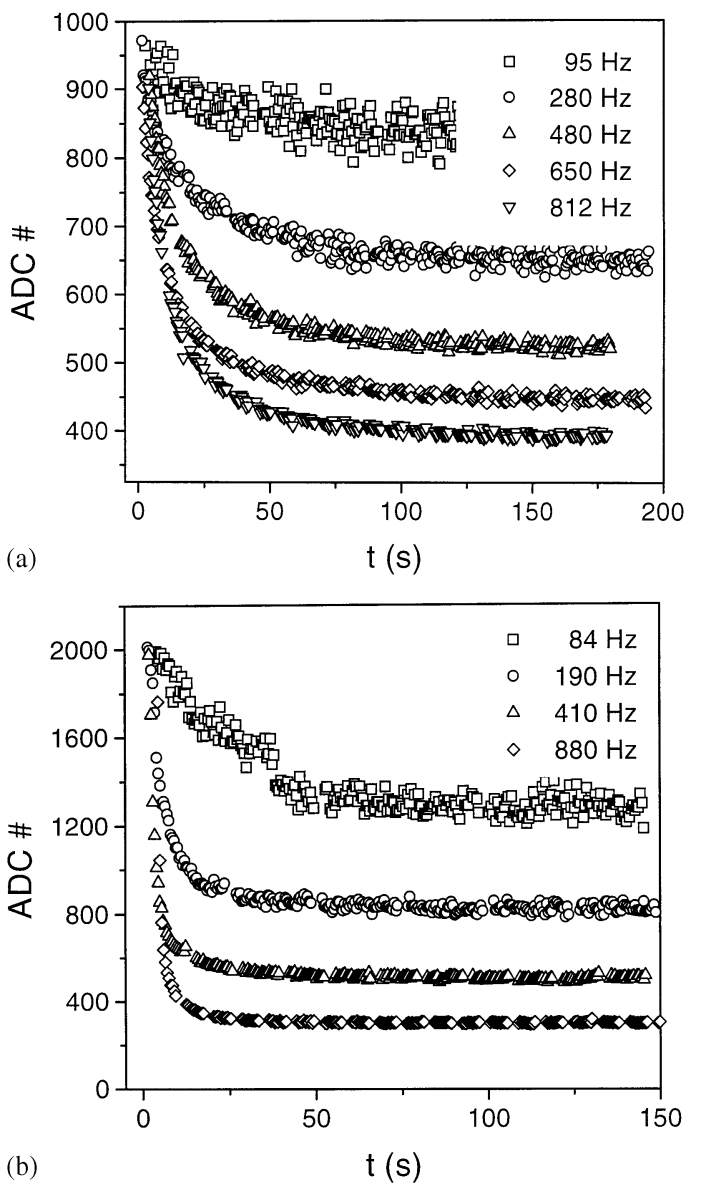

Fig. 4. Charge pulse height as a function of time, as measured for: (a) the cylindrical glass tube $\left(V_{0}=1700 \mathrm{~V}\right)$; (b) the parallel plate chamber $\left(V_{0}=-5250 \mathrm{~V}\right)$.

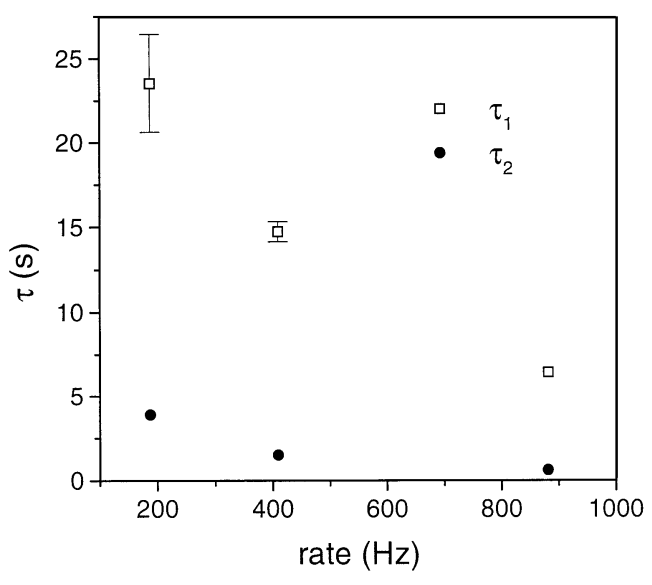

Fig. 5. Variation of the time decay constants with the counting rate, for the parallel plate chamber.

value, $I$, that corresponds to the current under equilibrium conditions. The variation of the pulse amplitude as a function of time is shown in Fig. 4 for both detectors under constant applied voltage and changing counting rate. Similar results were obtained for different applied voltages.

The fact that $i_{\mathrm{g}}$ tends to a constant value prevents us from applying the fitting procedure referred to in the previous section. Instead, an analysis of the data in terms of an exponential decay was attempted. It was found that for the lower current densities a simple exponential plus a constant term fits well the experimental data, whereas for higher currents a sum of two exponentials plus a constant term was needed. The time constants show a tendency to decrease with increasing counting rate (see Fig. 5) and are similar to those associated with the decay of the depolarisation current. However, in the electric measurements no variation of the time constants with the applied voltage was observed.

\section{Conclusions}

In the present work, measurements of the electric parameters of the glasses used were performed. It is also shown, with electric measurements, that these glasses feature a complex relaxation mechanism and, therefore, cannot be treated as equivalent to simple RC circuits. Accordingly, the time evolution 
of the amplitude of the charge signals in our resistive detectors is expected to be represented by a complex function. However, it was found that most of the experimental data can be fitted by a two exponential decay plus a constant term. Although the associated decay constants are not directly related to the fundamental processes, they are characteristic of a particular material and give an idea of how fast is the effective relaxation of the dielectric. These constants depend on the rate and may be larger up to a factor of 10 or of the order of the $\rho \varepsilon \varepsilon_{0}$ value. The influence of the counting rate, gain, electrode thickness, resistivity or other parameters on the time constants is still under investigation.

\section{Acknowledgements}

This work was supported in Portugal by JNICT (contracts CERN/FAE/1098/96 and CERN/FAE/1143/97), and in Brazil by FAPESP and $\mathrm{CNPq}$.

\section{References}

[1] E. Cerron Zeballos et al., Nucl. Instr. and Meth. A 367 (1995) 388.

[2] I. Crotty et al., Nucl. Instr. and Meth. A 337 (1994) 370.

[3] P. Fonte et al., Proc. IV Int. Workshop on Resistive Plate Chambers and Related Detectors, Scientifica Acta, Quaderni del Dottorato, Univ. of Pavia, to be published.

[4] I. Crotty et al., Nucl. Instr. and Meth. A 346 (1994) 107.

[5] M. Anelli et al., Nucl. Instr. and Meth. A 300 (1991) 572.

[6] D.F. Anderson et al., Nucl. Instr. and Meth. A 348 (1994) 324.

[7] M. Fraga et al., Proc. IV Int. Workshop on Resistive Plate Chambers and Related Detectors, Scientifica Acta, (Quaderni del Dottorato), Univ. of Pavia, to be published.

[8] Carmen C. Bueno et al., Nucl. Instr. and Meth. A 408 (1998) 496.

[9] D.G. Holloway, Physical Properties of Glass, London, 1973.

[10] S.K. Mendiratta, L.C. Costa, J. Non-Crystalline Solids 131-133 (1991) 990. 